\title{
Cerebral Gliomas Patterns at the General Hospital of Douala, Cameroon
}

\author{
Aurélien Ndoumbe ${ }^{1 *}$, Mathieu Motah1, Bea Jesse Ikango', Mireille Moumi², Amadou Fewou ${ }^{3}$, \\ Albert Mouelle Sone ${ }^{1}$ \\ ${ }^{1}$ Faculty of Medicine \& Pharmaceutical Sciences, University of Douala, Douala, Cameroon \\ ${ }^{2}$ Neurosurgeon, Service of Surgery, General Hospital of Douala, Douala, Cameroon \\ ${ }^{3}$ Pathology, Faculty of Medicine \& Biomedical Sciences, University of Yaoundé I. Head, Service of Pathology, General Hospital of \\ Douala, Douala, Cameroon \\ Email: *aurelien.ndoumbe@gmail.com, *aurelen@yahoo.fr
}

How to cite this paper: Ndoumbe, A., Motah, M., Ikango, B.J., Moumi, M., Fewou, A. and Sone, A.M. (2018) Cerebral Gliomas Patterns at the General Hospital of Douala, Cameroon. Open Journal of Modern Neurosurgery, 8, 389-405.

https://doi.org/10.4236/ojmn.2018.84035

Received: September 10, 2018

Accepted: October 15, 2018

Published: October 18, 2018

Copyright (C) 2018 by authors and Scientific Research Publishing Inc. This work is licensed under the Creative Commons Attribution International License (CC BY 4.0)

http://creativecommons.org/licenses/by/4.0/

\section{Open Access}

\begin{abstract}
The diagnosis and management of cerebral gliomas are challenging. The goal of this study was to evaluate the pattern of cerebral gliomas at the General Hospital Douala, Cameroon. A retrospective study was carried out over a period of 10 years. Non-glial lesions were not included. Data were analyzed with Epi info version 7.2.2.6 (Microsoft, Seattle, USA). 220 cases of brain tumors, comprising 56 gliomas (25.46\% of all brain tumors) were managed during the study period. The mean age of the patients was $42.93 \pm 16.90$ years. 35 cases $(62.5 \%)$ were males. Patients over 15 years of age represented 91.07\% $(n=51)$ of the cases. The most frequent presenting complaints were headache and vomiting with 52 cases $(92.86 \%)$ and 47 cases $(83.93 \%)$ respectively. Brain computed tomography was done for all patients. The most frequent histological type was astrocytoma with 22 cases (39.29\%) followed by glioblastoma with 20 cases $(35.71 \%)$. The most performed surgical technique was partial removal with 33 cases (58.93\%), followed by open biopsy with 14 cases $(25.00 \%)$. Radiotherapy was administered to 54 patients $(96.43 \%)$. The outcome ranged from full recovery in 8 cases $(14.29 \%)$, recovery with persisting symptoms, in 15 cases (26.79\%) and death in 26 cases (46.43\%). Seven patients $(12.50 \%)$ were lost to view. In this series, cerebral gliomas affected most often adult males. Most of them presented with signs of raised intracranial pressure. The outcome was poor with $46.43 \%$ of patients dying before 2 years after diagnosis.
\end{abstract}

\section{Keywords}

Cerebral Gliomas, Clinical Manifestations, Histological Nature, Management, Outcome 


\section{Introduction}

A tumor is a growth or "lump" made of cells that have begun to grow in an unusual way [1]. Cerebral gliomas (CG) are brain tumors that grow from glial cells [2]. Glial cells include astrocytes, oligodendrocytes, microglia, and ependymal cells [3]. The role of Glial cells is supporting of neuronal function and metabolism, the upholding of the blood-brain barrier and immune surveillance [4]. Astrocytes are Star-shaped cells providing support functions in the central nervous system [5]. Oligodendrocytes are cells that make the myelin sheath which covers and protects axons in the central nervous system [3]. Ependymal cells are cuboidal or columnar cells which line the brain ventricles and possess microvilli and cilia [5].

CG are considered as primary brain tumors, meaning that they originate from cells in the brain [1]. CG can be considered as either benign or malignant depending on their ability to invade other tissues [1]. CG can be divided into 3 main types: astrocytomas, oligodendrogliomas, and ependymomas. Gliomas comprise more than $80 \%$ of primary brain tumors [6], therefore, descriptive epidemiology about gliomas is often framed in the broader context of brain tumors as a whole. There is a tendency towards a higher incidence of gliomas in highly developed industrialized countries. Some studies indicate that Caucasians have a higher incidence than Africans or Asians [7] [8] [9]. Globally, the annual incidence of glial tumors in 2015 was estimated to be between 2.96 to 2.09 cases per 100,000 men and women respectively, with a higher rate in developed countries $(4.08 / 100,000$ persons-year) than developing countries $(2.4 / 100,000$ persons-year) [6].

The incidence of glial tumors in the United States of America is estimated to be about 11.2 cases per 100,000 persons annually. In France, the incidence of glial tumors annually is estimated to be about 7 cases per 100,000 persons-year for adults and 5 cases per 100,000 persons-year for children. The incidence of glial tumors in Northern Africa is estimated to be about 5 per 100,000 persons and in Sub-Saharan Africa, 0.8 cases per 100,000 persons [10].

In Cameroon as a whole, very little study has been carried out on BGs. However, studies carried out by Enow Orock et al. [11] on the incidence and trends of cancer in the Yaounde population estimated the incidence of BGs to be about 6.4 per 100,000 persons-year with a male:female ratio of about 1:1.5. The most common histological type found was astrocytomas (35\% of BTs), and ependymomas (7.5\% of BTs).

Clinically, the general presentation of brain tumors depends on the location, size of the tumor, and growth rate of the neoplasm [3]. Common presenting symptoms of brain tumors are symptoms of raised intracranial pressure, seizures and a progressive focal neurological deficit [12].

The management of glial tumors has evolved over the years in developed countries, with more sophisticated minimal invasive (stereotactic mass spectrometry, radio-neurosurgery) and post-invasive (immunohistochemistry) techniques other than those found in less developed countries being used. In less de- 
veloped countries, modern techniques available include; radiological imaging and pathological examination of the tumor mass. For radiological imaging, Brain CT scan and Magnetic Resonance Imagery are usually used.

The management of cerebral gliomas is multidisciplinary and disciplines involved include; neurosurgery, medical oncology, radiotherapy, medical imaging, neurologist and pathologists. The management of BG depends on the type of glioma and the grade of the tumor [13]. High grade tumors which are usually more invasive tend to be more resistant to treatment and therefore, have a poor prognosis [13]. The mortality rate due to gliomas in the United States of America is high with an average 5 -year survival rate being only about $35 \%$ of diagnosed patients [6].

This study was done in order to have a better understanding of cerebral gliomas in our setting.

\section{Patients and Methods}

It was a cross-sectional retrospective review of cerebral gliomas cases managed at the General Hospital Douala (GHD), Cameroon, over a period of ten years from January 2008 to December 2017.

Selection criteria were as follows: patients of any age with a definite diagnosis of cerebral glioma (CG) confirmed by pathological analysis. Available imaging and pathology data were mandatory for inclusion in the study. Patients treated for non-glial cerebral lesions and those with incomplete medical files were not included. The study had agreement from the institutional ethical committee.

Data were collected from patients' medical files at neurosurgery, neurology, oncology, radiology, radiotherapy and pathology units.

Variables such as age, gender, profession, marital status, previous exposure to ionizing radiations, history of traumatic brain injury, brain surgery, family history of CG, and exposure to pesticides were thoroughly reviewed. Clinical manifestations with thorough and concise neurological examination were collected, imaging data, pathology results, and management procedures (medical treatment, surgical procedure, radiation therapy and chemotherapy) also were gathered and analyzed. The pre and post-treatment Karnofsky [14] performance scale was determined (Table 1). The outcome of the patients was evaluated for mortality, survival, permanent disability and identification of prognosticators. The Kaplan-Meier survival curves were drawn. Patients who were still alive were contacted by the phone.

The statistical analysis was done with the software SPSS 20.0 for Windows ${ }^{\circ}$ [Microsoft, Seattle, USA]. The Fisher and the Chi-square tests were used. The statistical significance was set for $\mathrm{p}$ value $<0.05$.

\section{Results}

\subsection{Sociodemographic Data}

During the study period, 20,610 patients were hospitalized in the surgery and 
oncology units, $220(1.07 \%)$ of them were treated for brain tumors, $56(25.46 \%)$ of these brain tumors were confirmed as gliomas on pathology. Amongst patients with CG, 35 (62.5\%) were males and 21 (37.5\%) were females. Their mean age was $42.93 \pm 16.90$ years. Figure 1 gives the age distribution of patients with CG according to the gender.

\subsection{Clinical Data}

The most common presenting complaint were headache, vomiting, and motor weakness while altered consciousness, hemiparesis, and papilledema were the most common physical examination findings. Table 2 gives details of the clinical manifestations in patients with CG in this series.

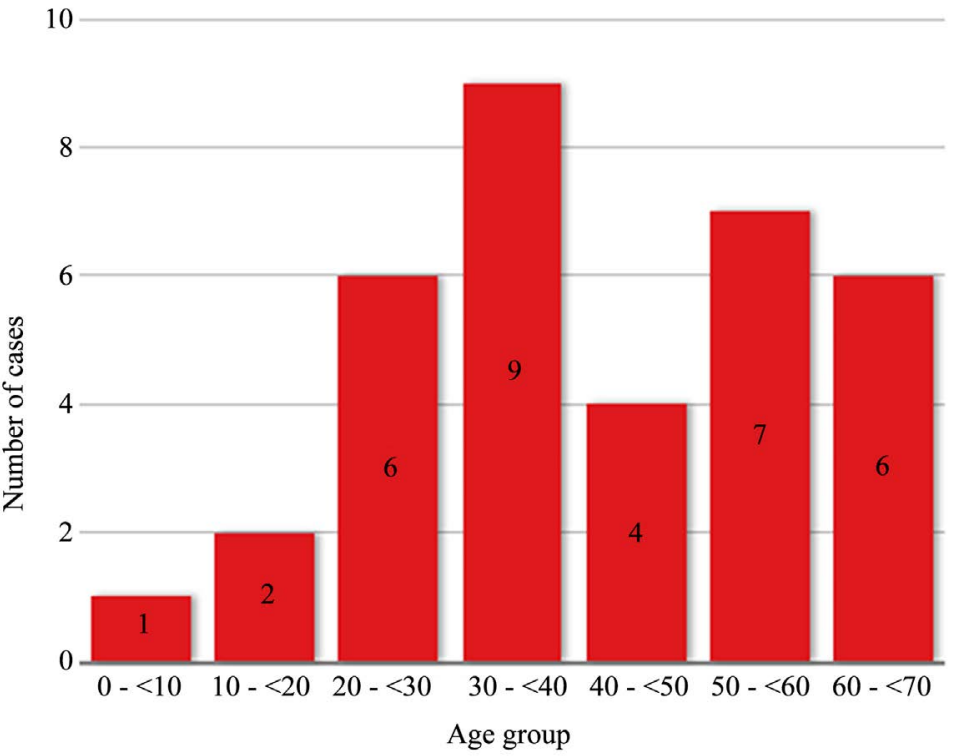

(a)

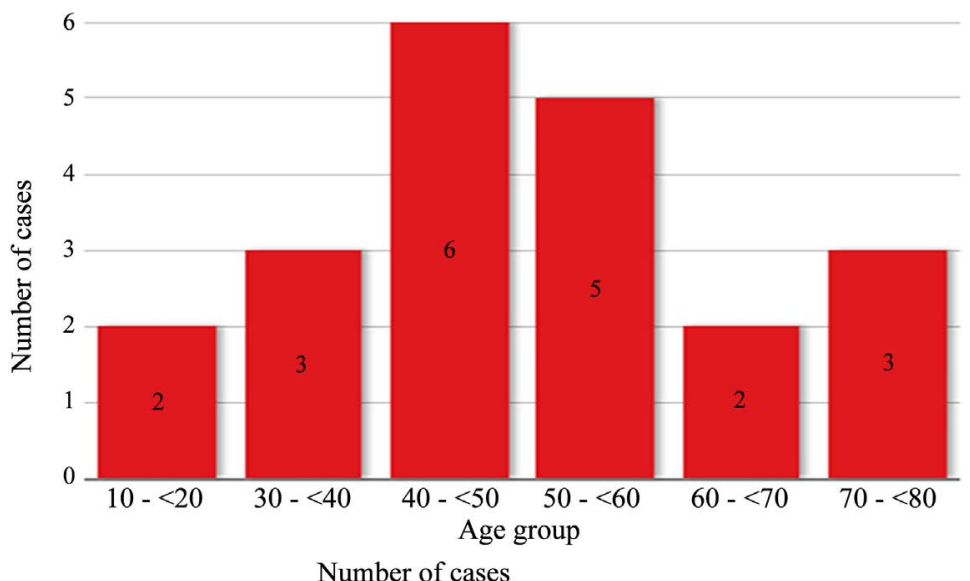

(b)

Figure 1. (a) Distribution of gliomas with respect to age group in males. The $30-39$ years age group had the highest frequency in males with 9 cases. (b) Distribution of gliomas with respect to age group in females. The $40-49$ years age group had the highest frequency in females with 6 cases. 
Table 1. Karnofsky performance scale [14].

100 Normal no complaints; no evidence of disease.

Able to carry on normal activity and to work; no special care needed. and care for most personal needs; varying amount of assistance needed.

Unable to care for self; requires equivalent of institutional or hospital care; disease may be progressing rapidly
90

70

50

40

30

20

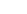

10 Able to carry on normal activity;
minor signs or symptoms of disease.

Normal activity with effort; some signs or symptoms of disease

Cares for self; unable to carry on normal activity or to do active work.

Requires occasional assistance, but is able to care for most of his personal needs.

Requires considerable assistance and frequent medical care.

Disabled; requires special care and assistance.

Severely disabled; hospital admission is indicated although death not imminent.

Very sick; hospital admission necessary; active supportive treatment necessary.

Moribund; fatal processes progressing rapidly. Dead

Table 2. Symptoms and signs of patients with cerebral gliomas.

\begin{tabular}{|c|c|c|}
\hline COMPLAINT & FREQUENCY & PERCENT \\
\hline Headache & 52 & $92.86 \%$ \\
\hline Vomiting & 47 & $83.93 \%$ \\
\hline Hemiparesis & 37 & $66.07 \%$ \\
\hline Seizures & 21 & $37.50 \%$ \\
\hline Vision disturbance & 12 & $21.43 \%$ \\
\hline Behavioral impairment & 11 & $19.64 \%$ \\
\hline Aphasia & 11 & $19.64 \%$ \\
\hline Vertigo & 8 & $14.29 \%$ \\
\hline Equilibrium disorder & 4 & $7.14 \%$ \\
\hline Hypoacousia & 4 & $7.14 \%$ \\
\hline Dysphonia & 1 & $1.79 \%$ \\
\hline PHYSICAL FINDINGS & FREQUENCY & PERCENT \\
\hline Altered consciousness & 48 & $85.71 \%$ \\
\hline Hemiparesis & 37 & $66.07 \%$ \\
\hline Papillary edema & 33 & $58.93 \%$ \\
\hline Hyper-reflexia & 19 & $33.93 \%$ \\
\hline Diminished visual acuity & 14 & $25.00 \%$ \\
\hline Cognitive disorder & 13 & $23.21 \%$ \\
\hline Aphasia & 9 & $16.07 \%$ \\
\hline Diplopia & 9 & $16.07 \%$ \\
\hline Memory disorder & 7 & $12.50 \%$ \\
\hline Co-ordination disorder & 7 & $12.50 \%$ \\
\hline Cranial nerve palsy & 7 & $12.50 \%$ \\
\hline Abnormal gait & 5 & $8.93 \%$ \\
\hline Hypo-aesthesia & 3 & $5.36 \%$ \\
\hline Exophthalmos & 1 & $1.79 \%$ \\
\hline
\end{tabular}

Symptoms and signs of intracranial hypertension were the mostcommonclinical manifestations. 


\subsection{Radiological and Pathology Data}

A brain CT scan was done for all patients and some had brain MRI. A space occupying lesion was confirmed in all cases. Table 3 summarizes the CT findings. According to WHO grading, the CG was grade I in 4 cases, grade II in 20 cases, grade III in 12 cases, and grade IV in 20 cases respectively. Astrocytomas were the most frequent histologic type encountered with 42 (75\%) cases out of 56 . Seventy-five percent of glioblastoma cases occurred in patients aged 40 years and above, while $45.45 \%$ of oligodendrogliomas concerned patients between 30 and 39 years. The age distribution of astrocytomas was more even with $50 \%$ occurring in patients less than 40 years old and $50 \%$ in those aged 40 years or more. Ependymomas occurred in young or old patients, that is, before 30 years or after 60 years, no case was recorded in patients aged between 30 and 59 years. Figure 2 gives detail on the histological nature of the CG of the series.

\subsection{Management Data}

All patients had symptomatic treatment. It consisted in analgesics in all cases with paracetamol given alone or in combination to 50 patients, pregabalin to 32 and tramadol to 11 . Corticosteroids were given to 54 patients consisting in oral or IV methyl-prednisolone in 53 cases. Anticonvulsants were given to 38 patients, phenobarbital was given alone or in combination to 18 patients, and sodium valproate toten. All patients had a surgical procedure. It consisted to open biopsy in 14 cases, partial resection in 41 cases, and total removal in only one case. Five patients had additional ventriculoperitoneal shunt for associated obstructive hydrocephalus. Fifty four $(96.43 \%)$ patients underwent radiation therapy after surgery. The dosage of radiation with respect to histological nature ranged from 10 to 60 Grays. Table 4 gives details of the radiotherapy modalities according to tumor type. No patient had chemotherapy.

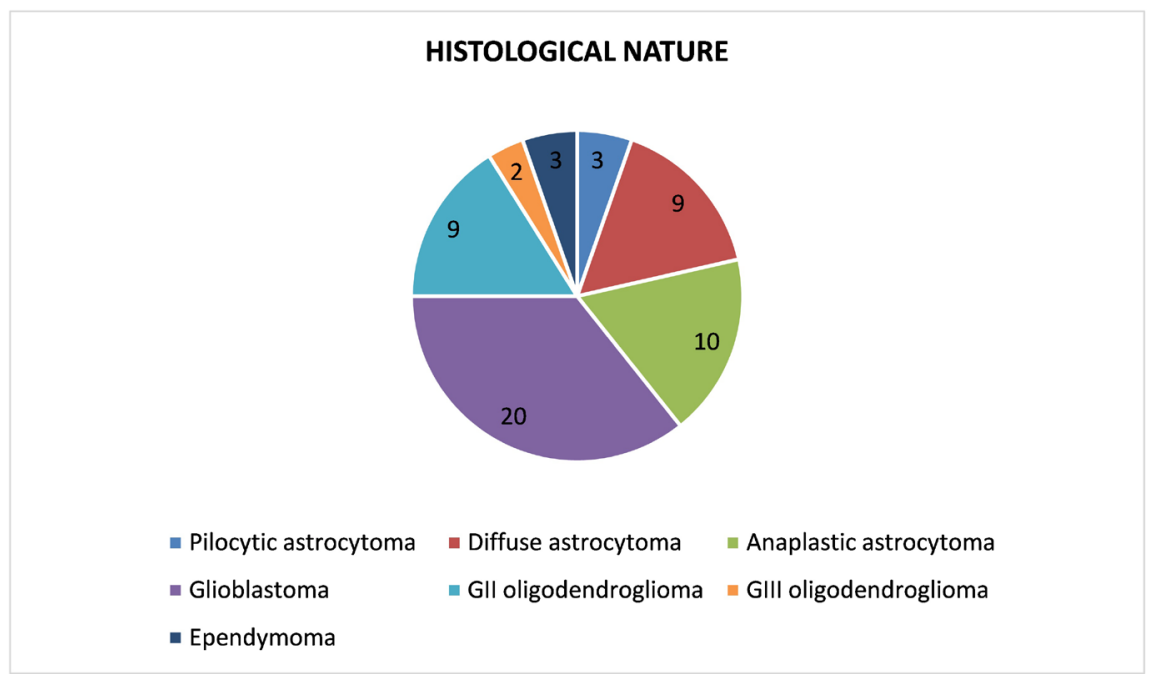

Figure 2. Histological nature of the cerebral gliomas. The glioblastoma was the mostfrequent type followed by other astrocytomas. 
Table 3. Brain computed tomography findings.

\begin{tabular}{ccc}
\hline BRAIN CT SCAN FINDING & FREQUENCY & PERCENTAGE \\
\hline LOCATION OF LESION. & 33 & $58.93 \%$ \\
Left side & 23 & $41.07 \%$ \\
Right side & 28 & $50.00 \%$ \\
Frontal lobe & 16 & $28.57 \%$ \\
Parietal lobe & 09 & $16.07 \%$ \\
Temporal lobe & 08 & $14.29 \%$ \\
Occipital lobe & 10 & $17.86 \%$ \\
Posterior fossa & & \\
NUMBER OF LESIONS. & 41 & $73.21 \%$ \\
Isolated lesion & 12 & $21.43 \%$ \\
Multiple lesions & & \\
LESION SURROUNDINGS. & 46 & $82.14 \%$ \\
Irregular & 07 & $12.50 \%$ \\
Circumscribed & & $89.30 \%$ \\
DENSITY OF LESION. & 50 & $07.14 \%$ \\
Hypodense lesions & 04 & $01.79 \%$ \\
Isodense lesions & 01 & $82.14 \%$ \\
Hyperdense lesions & & $73.21 \%$ \\
ASSOCIATED SIGNS. & 46 & $48.21 \%$ \\
Perilesional edema & 41 & $44.64 \%$ \\
Shifting & 27 & \\
Necrosis & 25 & \\
Hydrocephalus & & \\
Calcification & & \\
\hline
\end{tabular}

CT: computed tomography. Most gliomas were located on the left cerebral hemisphere and the frontal lobe was the most common site involved. The CT scans showed hypodense lesions surrounded with edema in most of the cases.

Table 4. Radiotherapy according to tumor type.

\begin{tabular}{ccc}
\hline DOSAGE (Gray) & FREQUENCY & PERCENTAGE \\
\hline Glioblastoma. & 5 & $26.32 \%$ \\
$30-39 \mathrm{~Gy}$ & 1 & $5.26 \%$ \\
$40-49 \mathrm{~Gy}$ & 10 & $52.63 \%$ \\
$50-59 \mathrm{~Gy}$ & 3 & $15.79 \%$ \\
$>60 \mathrm{~Gy}$ & & \\
Astrocytoma. & 4 & $19.05 \%$ \\
$20-29 \mathrm{~Gy}$ & 1 & $4.76 \%$ \\
$30-39 \mathrm{~Gy}$ & 10 & $47.62 \%$ \\
$50-59 \mathrm{~Gy}$ & 6 & $28.57 \%$ \\
$>60 \mathrm{~Gy}$ & & \\
Oligodendroglioma. & 1 & $9.09 \%$ \\
$10-19 \mathrm{~Gy}$ & 1 & $9.09 \%$ \\
$20-29 \mathrm{~Gy}$ & 2 & $18.18 \%$ \\
$30-39 \mathrm{~Gy}$ & 7 & $63.64 \%$ \\
$50-59 \mathrm{~Gy}$ & & $33.33 \%$ \\
Ependymoma. & 1 & $33.33 \%$ \\
$10-19 \mathrm{~Gy}$ & 1 & $33.33 \%$ \\
$20-29 \mathrm{~Gy}$ & 1 & \\
$50-59 \mathrm{~Gy}$ & &
\end{tabular}

Gy: Grays. Most patients received whole brain irradiation of 50 to 59 Grays. 


\subsection{Outcome Data}

Table 5 shows the pre-treatment Karnofsky performance scores of the patients. For patients with ultimate diagnosis of gliobastoma, only one out of 20 (5\%) was able to carry on normal activity with effort, most of the remainders were disabled or required considerable assistance predicting their subsequent poor prognosis. For patients with other astrocytomas, 12/22 (54.54\%) were able to carry on normal activity, without effort for most of them; eight (36.36\%) were able to care for self with or without occasional assistance, and only two (9\%) were disabled. For patients with oligodendrogliomas, $81.81 \%(9 / 11)$ were able to carry on normal activity, without effort for most of them, while two (18.18\%) needed considerable assistance. Therefore, the pre-treatment Karnofsky performance score was strongly predictive for the outcome. Twenty-six out of 56 (46.42\%) patients died during the study period, 18 of them had a glioblastoma and 8 astrocytoma. No recorded death occurred in patients with oligodendrogliomas or ependymomas. Therefore, the mortality prevalence was $90 \%(18 / 20)$ and $36.36 \%(8 / 22)$ for glioblastomas and astrocytomas respectively. Figure 3 shows the Kaplan-Meier survival curve for astrocytomas and glioblastomas. Concerning the functional outcome, 8 patients recovered without signs of disease, 15 had persisting symptoms, and 7 were unreachable. The persisting symptoms and signs were as follows: visual disturbances, 6 cases, hemiparesis and seizures, 4 cases each, and aphasia, 3 cases. An illustrative clinical case is shown on Figure 4.

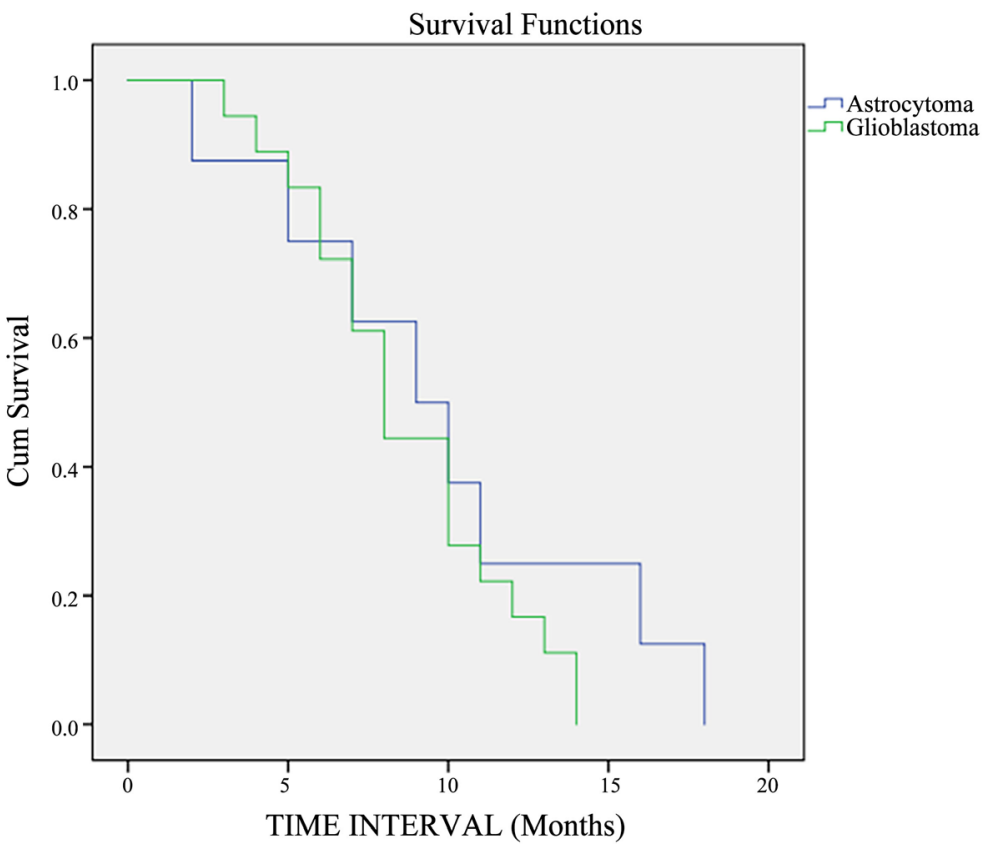

Figure 3. Kaplan Meier curve for time interval between diagnosis and death. The mean survival time interval in gliobastoma patients was about 7 months after diagnosis while the mean survival time interval in astrocytoma patients was about 10 months after diagnosis. The log-rank test score was 0.995 and a $\mathrm{P}$ value of $0.319(>0.05)$ indicating that there is no statistical significance between the two groups. 

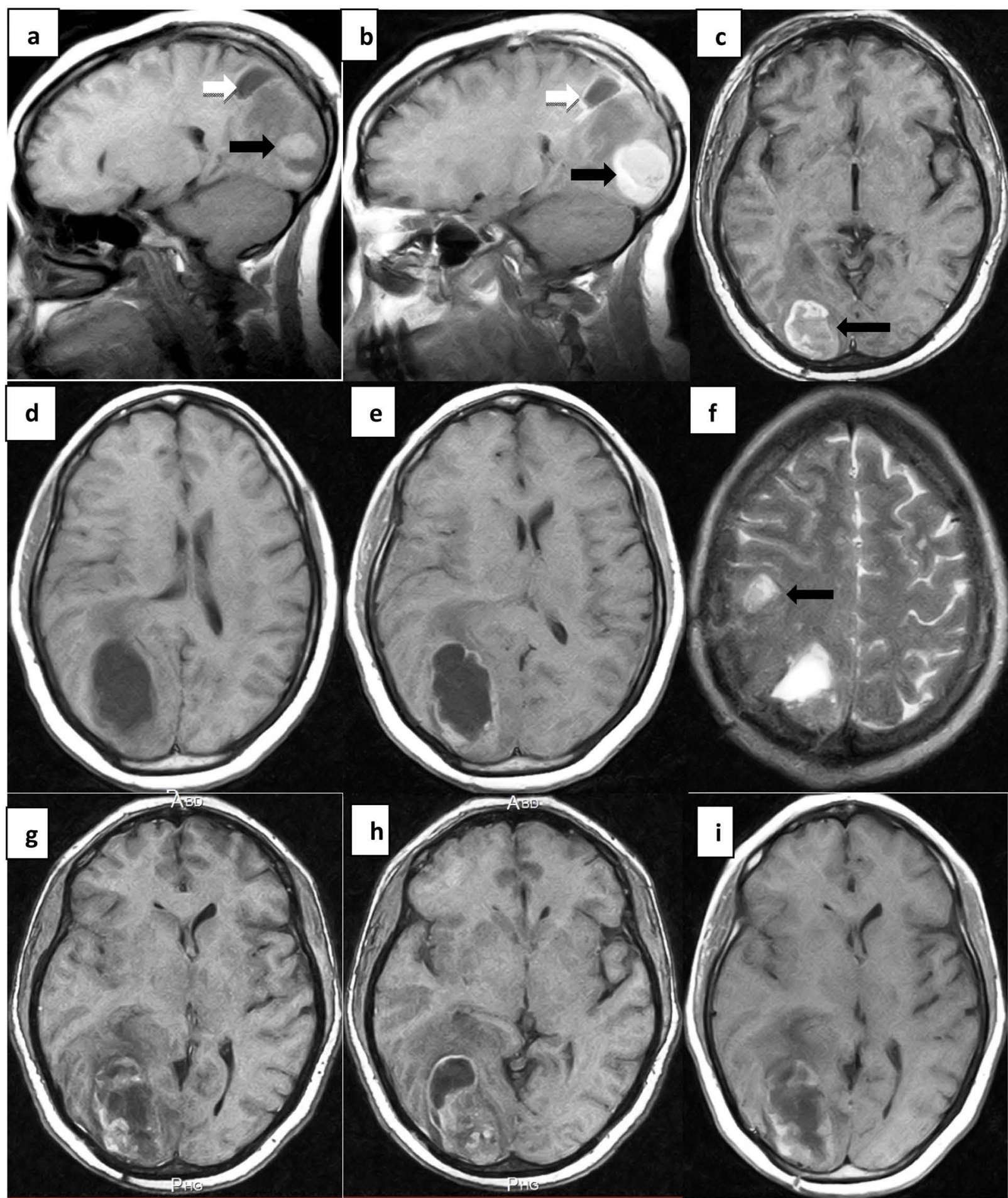

Figure 4. Clinical case. This 42 years old woman presented with symptoms of intracranial hypertension. MRI showed a right parietal lesion. Gross total removal was achieved and pathology was in favor of WHO grade I microcystic meningioma. No further action was taken. The patient came three months later for recurrence of symptoms. Control MRI showed right occipital lobe lesion (black arrow), not present on the first examen and no tumor recurrence at the first operative site (white arrow), a, b, c. An open biopsy was done and pathology was in favor of WHO grade IV astrocytoma (glioblastoma multiforme). Radiotherapy was started. But, control MRI a month later showed progression of tumor (d, e) with appearance of a third lesion on the right frontal lobe (f, black arrow). $\mathbf{g}, \mathbf{h}$, and $\mathbf{i}$ show heterogeneity of the tumor. The patient died few months later in spite of radiotherapy. 
Table 5. Pre-treatment Karnofsky performance score with respect to the histological nature.

\begin{tabular}{ccc}
\hline SCORE & FREQUENCY & PERCENT \\
\hline Astrocytoma & 2 & $9.09 \%$ \\
40 & 5 & $22.73 \%$ \\
60 & 3 & $13.64 \%$ \\
70 & 3 & $13.64 \%$ \\
80 & 4 & $18.18 \%$ \\
90 & 5 & $22.73 \%$ \\
100 & & \\
Oligodendroglioma & 2 & $18.18 \%$ \\
50 & 2 & $18.18 \%$ \\
80 & 5 & $45.45 \%$ \\
90 & 2 & $18.18 \%$ \\
100 & & \\
Ependymoma & 1 & $33.33 \%$ \\
60 & 1 & $33.33 \%$ \\
80 & 1 & $33.33 \%$ \\
100 & & \\
Glioblastoma & 4 & $20.00 \%$ \\
30 & 4 & $20.00 \%$ \\
40 & 5 & $25.00 \%$ \\
50 & 1 & $5.00 \%$ \\
60 & 5 & $25.00 \%$ \\
70 & 1 & $5.00 \%$ \\
80 & & \\
\hline
\end{tabular}

The pretreatment Karnofsky performance scores were strongly bond with type of gliomas and the subsequent outcome.

\section{Discussion}

The aim of this study was to have a global view on brain gliomas patterns and management in our setting. The limitations of this study were as follows: it was retrospective cross-sectional analysis, therefore, gathering of data was not perfect; immunochemistry was not available and accurate categorization of lesions such as astrocytomas was not feasible.

\subsection{Epidemiology}

Gliomas represent more than $80 \%$ of primary brain tumors [13], but incidence varies by histology, race, and sex. Astrocytomas form the largest group of gliomas (>75\%), glioblastoma multiforme (GBM) being the most frequent and the most malignant type [15]. Glioblastomas account for about $60 \%-70 \%$ of all gliomas and about $17 \%$ of all primary brain tumors. A majority of glioblastoma patients $(87 \%)$ are aged $55-87$ years. Oligodendrogliomas account for about $25 \%-33 \%$ of gliomas. They occur mostly in middle-aged adults, usually between the $4^{\text {th }}$ and $5^{\text {th }}$ decades of life. Ependymomas are a rare type of glial tumors, most intracranial ependymomas (36\% - 60\%) occur in children [16].

Cerebral gliomas represented $25 \%$ of brain tumors managed at our hospital. Astrocytomas accounted for $75 \%$ of these gliomas. The results of this series showed that, astrocytomas other than GBM was the most frequent age-adjusted 
histological type with 22 cases (39.29\%), followed by grade IV astrocytoma or GBM with 20 cases (35.71\%), oligodendrogliomas with 11 cases (19.64\%) and ependymomas were less frequent with 3 cases (5.36\%) recorded. GBM was most frequent in patients between 50 and 59 years with 7 cases $(35.00 \%)$ followed by the 40 to 49 years age group with 5 cases $(25.00 \%)$. These results are similar to those reported in other studies. Astrocytomas were most frequent in patients between 50 and 69 years with 10 cases (40.00\%). Oligodendrogliomas were most frequent in patients between 30 to 39 years. Ependymomas were seen in patients aged less than 30 or above 60 . Studies carried out in the U.S.A in 2011 showed that, the age-adjusted rate per 100,000 persons-year for glioblastoma is 3.19 compared with 0.12 for anaplastic oligodendrogliomas and 0.11 for protoplasmic/fibrillary astrocytomas [15]. The lack of immunohistochemistry in our setting did not allow the specific categorization of astrocytomas subtypes.

This series also showed that the age-adjusted frequency of cerebral gliomas was higher in males with a frequency of 35 cases (62.5\%) against 21 cases $(37.5 \%)$ in females, $(\mathrm{SR}, 1.5: 1)$. In males, the $4^{\text {th }}$ and $6^{\text {th }}$ decades were the most involved with nine $(25.71 \%)$ and seven cases $(20.00 \%)$ respectively. In females, the most frequent age groups were $5^{\text {th }}$ and $6^{\text {th }}$ decades $(28.27 \%$; and $23.81 \%$ of cases). Astrocytomas and GBM were the most common gliomas both in males and females in the present study. In 2005; the estimated worldwide incidence of cerebral gliomaswas 3.7 per 100,000 persons-year in males and 2.6 per 100,000 person-years in females [17]. In 2011, studies carried out in the U.S.A estimated, the incidence rate of glioblastoma was about 3.99 per 100,000 persons-year in males against 2.53 per 100,000 persons-year in females. These incidences are similar to those in our series given that the incidence in males is higher than that in females [15].

Developed countries tend to have higher rates (incidence rate of 5.8 per 100,000 persons-year for men and 4.1 per 100,000 persons-year for females), than less developed countries (incidence rate of 3.0 per 100,000 persons-year for males and 2.1 per 100,000 persons-year for females) possibly reflecting less access to modern medical facilities [15], [17]. In contrast, studies carried out in Japan in 2011 showed that the incidence rate for cerebral gliomas, was relatively low (2.5 per 100,000 persons-year) [18].

\subsection{Clinical Manifestations}

The most common presentation of brain tumors is progressive neurologic deficit (68\%), usually motor weakness (45\%). Patients with primary brain tumors can present with generalized or focal signs and symptoms [16]. Typically, generalized symptoms occur later in the disease pathogenesis as the tumor grows and causes increased intracranial pressure leading to headache, seizure, nausea, vomiting, and altered mental function [16]. Focal symptoms such as focal neurological deficit reflect the intracranial location of the tumor. Roughly $77 \%$ of all patients with primary brain tumors report a dull tension-type headache that can 
persist for more than six months in $50 \%$ of patients. Although headache is the most common initial presenting symptom, other symptoms are often associated with headaches including seizures in $50 \%$ of patients, visual disturbances in $40 \%$ of patients, and nausea and vomiting in $38 \%$ of patients. Seizures are common in patients with brain tumors with $15 \%-95 \%$ of patients presenting with at least one seizure during the course of their illness [16].

In this series, the most frequent presenting complaints were, headache (92.86\%), vomiting (83.93\%), hemiparesis (66.07\%) and seizures $(37.50 \%$ of cases). Moderately frequent symptoms included; vision disturbances $(21.43 \%)$, behavioral impairment (19.64\%) and vertigo (14.29\%). Less frequent symptoms were; equilibrium imbalance with (7.14\%), hypoacousia (7.14\%) and dysphonia (1.79\%). Signs of raised ICP were present in most patients including: altered state of consciousness, 48 cases (85.71\%); headache, 52 cases (92.86\%); vomiting, 47 cases (83.93\%); and vision disorders such as papilledema, 33 cases $(58.93 \%)$, diminished visual acuity, 14 cases (25.00\%), and diplopia, 9 cases (16.07\%).

Hemiparesis was present 37 cases (66.07\%) with exaggerated deep tendon reflexes in 19 (33.93\%) of them. Co-ordination was impaired in $12.50 \%$ of the patients, $8.93 \%$ had abnormal gait, $14.29 \%$ a vertigo, $23.21 \%$ cognitive impairment, $16.07 \%$ had aphasia, and the cranial nerves were involved in $12.50 \%$ of the cases.

According to a study carried out at the National Hospital for Neurology and Neurosurgery at Queen Square London, on a large case record review of initial symptoms experienced by 653 glioma patients between 1955 and 1975 found that patients presented with neurological problems such as seizures (54\%), headache $(71 \%)$, mental change (52\%), and hemiparesis $(43 \%)$ by the time of diagnosis [19]. These results are similar to those obtained in this series.

\section{Imaging}

The scope of neuroradiology tumor imaging has continued to evolve, within the past decades imaging has moved from indirect diagnosis of lesion using cerebral angiography to precise lesion localization using multi planar CT scan or MRI and to lesion diagnosis using stereotactic mass spectrometry which can reveal a lesion's molecular composition [16]. Continued advancements have furthered the imaging role to include surveillance of treatment response and lesion recurrence. A comparison of between the CT scan and MRI reflects that while CT scan has many advantages (faster, cheaper and more readily available), MRI remains the modality of choice given its superior soft tissue contrast resolution [16].

The presentation of the lesion on imagery depends on the imaging technique used and the histological nature of the tumor [12].

In this series, the brain CT scan was done in all patients, but only few did MRI. This is because the CT scan is, cheaper and more readily available in our setting. The brain CT scan findings were as follows: the left hemisphere was involved in $58.93 \%$ of cases, and the frontal lobe in $50.00 \%$. The lesions were iso- 
lated in $73.21 \%$. The contours were irregular in $82.14 \%$. The gliomas were hypo-dense in $89.30 \%$, with surrounding edema in $82.14 \%$ of the cases, brain shifting was present in $73.21 \%$ and necrosis in $48.21 \%$. Most low grade gliomas are hypo intense on T1WI MRI, and T2WI shows high signal changes that extend beyond the tumor volume. Most low-grade gliomas do not enhance on CT or MRI, although up to $40 \%$ do; some malignant gliomas do not enhance. Glioblastoma multiforme typically shows ring enhancement on CT, with low density necrotic center, and enhancing ring corresponding to tumor cells.

\section{Management}

The management of cerebral gliomas has many aspects which include: symptoms relief, tissue diagnosis, restoring cerebrospinal fluid flow and decompressing adjacent neural structures, radiation therapy and chemotherapy. Non-surgical alternatives such as radiosurgery can be contemplated when an invasive procedure is precluded [16].

Symptomatic treatment is achieved by medications such as corticosteroids, analgesics, anti-epileptics and anxiolytics.

The tissue diagnosis can be made after surgical techniques such as open or stereotactic biopsy, partialor gross total resection. Gross total removal of the lesion provides the best clinical outcomes. When risk of surgery is too high or if gross total resection cannot be performed, consideration for chemotherapy and radiotherapy can be given [16]. Recent developments make use of radio-neurosurgery which is a highly advanced radiation technology that permits maximum dose delivery within the target while minimizing dose to the surrounding healthy brain tissue [16].

Chemotherapy regimens usually consist of a specific number of cycles given over a set period of time. The main hindrance to effective chemotherapy use for gliomas is the blood brain barrier which prevents most drugs from getting to the brain parenchyma. However, some drugs still go through the blood-brain barrier. These drugs include: nitrosoureas such as carmustine or, lomustine, nimustine; alkylating (methylating) agents like temozolomide and procarbazine; carboplatin and cispaltin; nitrogen mustards as cyclophosphamide, isofamide and cytoxan; and vinca alkaloids such as Vincristine, vinblastine, paclitaxel; and so on.

The chemotherapy regimens used for treatment of CG are well established and usually begin with PCV (Procarbazine, CCNU and Vincristine). Temozolomide, a chemotherapy agent administered per os can also be used in the place of PCV. Its advantages are that it is easy to administer, it has lesser adverse effects and it is very efficient [20], [21]. Chemotherapy is usually reserved for tumor progression. The above regimens often stabilize tumor growth.

Concerning radiotherapy, the dose of irradiation for intracranial tumors varies (50 to 60 Grays given in a period of 5 to 6 weeks, divided as 2 Grays per session and 5 sessions per week). This dosage can however be modified with respect 
to the histological type [22]. Whole brain radiotherapy has not proven superior to focal irradiation in increasing survival.

The management of a patient with cerebral glioma depends on the histological nature of the tumor, the age of the patient and the Karnofsky score [1].

The options for managing a low-grade glioma include surgery and radiotherapy, either alone or combined [1]. Surgical biopsy or partial resection is recommended in almost all cases to establish the diagnosis. Surgery is also indicated for pilocytic astrocytomas, when herniation threatens from large tumors or tumors cysts, in case of CSF flow obstruction, to help seizure control, in smaller tumor which are less aggressive and in attempting to delay adjuvant therapy and its side-effects.

The options for managing high-grade gliomas include surgery, radiotherapy and chemotherapy usually two or three combined [1]. Cytoreductive surgery followed by external beam radiation is the standard treatment for malignant astrocytomas.

The surgical techniques usually used include biopsy, shunting, incomplete or complete resection of the lesion.

Chemotherapy is indicated in low grade gliomas (grade I and II) in case of a residual of the initial tumor.

Chemotherapy is recommended for all patients with high-grade gliomas (grade III and IV). It should begin at the same time as radiotherapy and continue for six months after radiotherapy.

In this series, all the patients received symptomatic treatment with analgesics, corticosteroids, anti-epileptics. The most frequent surgical technique performed was partial resection, followed by open biopsy. This can be explained by the fact that gliomas are usually infiltrating and complete resection usually impossible and risky, therefore radiotherapy is usually required after partial removal and biopsy. Patients with CSF obstruction had ventricular-peritoneal shunting besides tumor resection. Radiotherapy was administered to $96.43 \%$ of the patients. The dosage of radiation with respect to histological nature ranged from 10 to 60 Grays. The dosage of radiation varied from the norm because of avoidance of adverse effects of radiotherapy such as necrosis and cerebral atrophy which are difficult to manage in resource limited environments.

\section{Outcome}

The outcome of glioma patients depends on the interval between onset and treatment, the histological nature of the tumor and the treatment administered. The independent factors that statistically affect longevity are: patient age, histological features and performance status at presentation. The patient age is the most significant prognosticator, younger patients having better outcomes [23].

In the present series, of the eight (14.29\%) patients who recovered fully, five of them $(62.50 \%)$ had astrocytomas. For 15 patients who recovered partially, 14 (93.33\%) had low-grade astrocytomasor oligodendrogliomas with 7 cases each. It 
is noticeable that most patients with astrocytomas and oligodendrogliomas had high performance (Karnofsky) scores at presentation. For the 26 patients who died, 18 had GBM and the remainders anaplastic astrocytomas. These patients also presented with the lowest performance scores. No death was recorded in patients with low-grade gliomas no matter the histologic type. Therefore, we can conclud that the outcome of our patients was strongly influenced by the tumor type and neurological status before treatment.

Lamborn et al. [6] surveyed 832 GBM patients enrolled into prospective clinical trials in order to identify potential risk groups. From their analysis, they identified four risk groups in which the two lower risk groups included patients under the age of 40 with the lowest overall risk group being young patients with frontal lobe tumors only. The intermediate risk group included patients aged 40 - 65 with KPS > 70 who underwent subtotal or total resection of the tumor. Finally, the highest risk group included patients over the age of 65 or patients aged 40 - 65 with either KPS $<80$ or who only underwent biopsy. The results of this study are similar to those in our study in which we had a poorer prognosis in patients with a Karnofsky score less than 70 .

According to results of a study published by the Clinical Oncology Society of Australia in 2011, in people with low-grade oligodendrogliomas, median survival time after diagnosis was seven years. This means that half of these people survive for more than seven years. For people with high-grade oligodendrogliomas, median survival time after diagnosis was 11 - 14 months. This means that half of these people survive for more than 14 months [1]. People with high-grade astrocytomas generally have a low chance of survival. On average: about 1 in 3 (36\%) patients survive two years after the diagnosis; about 1 in $4(28 \%)$ patients survive five years after the diagnosis. Among people with a grade IV astrocytoma (the most malignant form), about 1 in 4 (27\%) who receive treatment with both radiotherapy and chemotherapy survives two years after the diagnosis [1]. These results are similar to those in our series in which, the time interval between diagnosis and death ranged from a few weeks to 20 months. Amongst patients with glioblastoma, the most frequent survival time interval was 6 to 8 months with 7 cases (38.89\%), followed by 9 to 11 months with 4 cases (22.22\%) and 12 to 14 months with 4 cases (22.22\%). Amongst patients with Astrocytoma, the most frequent survival time interval was 9 to 11 months with 3 cases (37.50\%).

\section{Conclusion}

The prevalence of cerebral gliomas for brain tumors managed at the General Hospital Douala was $25.46 \%$. Nine patients out of ten were adults, two-thirds were males. Most patients presented with intracranial hypertension and headache, altered consciousness, and vomiting were the most frequent clinical manifestations. Brain CT scanning was the most accessible imaging tool and it was done in all patients. Symptomatic treatment was administered to most patients and consisted in analgesics in all cases, corticosteroids in all but two cases, and 
anti-epileptics in two-thirds of the patients. All patients were operated most often for histological diagnosis purpose. The most performed surgical procedure was partial resection of the tumor. External beam whole brain irradiation was administered to all but two patients. Seventy-five per cent of the gliomas were astrocytomas, and glioblastoma (grade IV astrocytoma) was the most common type. The prognosis was poor since almost half of the patients died before two years after the diagnosis. All deaths concerned patients with glioblastomas or anaplastic astrocytomas, and death prevalence in patients with GBM was $90 \%$. The prognosis was strongly correlated with the histologic type, the grade of the glioma and the pre-treatment Karnofsky performance score which in turn was bond with the nature of the neoplasm. Patients with grade IV and grade III gliomas had the poorest performance scores at presentation and the worst outcome after treatment.

\section{Acknowledgements}

We thank Miss NDIMBA Erica for her help with the corrections.

\section{Conflicts of Interest}

The authors declare no conflicts of interest regarding the publication of this paper.

\section{References}

[1] Cancer Council Australia; Adult Gliomas (Astrocytomas and Oligodendrogliomas). http://www.cancer.org.au/clinicalguidelines

[2] Christopher, M. (2016) Glioma Brain Tumors. http://www.abta.org

[3] National Cancer Institute of the United States (2017) What You Need to Know about Brain Tumors. https://www.cancer.gov/

[4] Wagemakers, M. (2015) Angiogenesis and Angiopoietins in Human Gliomas. http://www.rug.nl/research/portal

[5] Siegel, A. and Sapru, H. (2010) Essential Neuroscience. Lippincott Williams and Wilkins, Philadelpia USA, Vol. 5, 380.

[6] CBTRUS (2015) Statistical Report: Primary Brain Tumors in the United States, 1998-2002. Central Brain Tumor Registry of the United States, Hinsdale, IL.

[7] Ohgaki, H. and Kleihues, P. (2005) Epidemiology and Etiology of Gliomas. Acta Neuropatholy, 109, 93-108. https://doi.org/10.1007/s00401-005-0991-y

[8] Jordan, C., Guzman, T. and Noble, M. (2006) Cancer Stem Cells. The New England Journal of Medicine, 355, 1253-1261. https://doi.org/10.1056/NEJMra061808

[9] Sayegh, E., Aranda, D., Kim, J. and Parsa, A. (2014) Prognosis by Tumor Location in Adults with Intracranial Ependymomas. Journal of Clinical Neuroscience, 21, 2096-2101. https://doi.org/10.1016/j.jocn.2014.05.011

[10] Aschoff, A., Kremer, P., Hashemi, B. and Kunze, S. (1999) The Scientific History of Hydrocephalus and Its Treatment. Neurosurgery Review, 22, 67-95. https://doi.org/10.1007/s101430050035

[11] Enow, O., Enoh, N. and Verla, T. (2015) Brain Tumors: Clinicopathological Aspects of 40 Cases in Cameroon and the Review of Literature. African Journal of Integrated Health, 5, 18-23. 
[12] Lain, W. and Graham, L. (2005) Essential Neurology. Blackwell Publishing, Massachusetts, USA, Vol. 4, 48-53.

[13] Jennifer, M. (2011) New Carriers for Invasive Glioma: Georgia Institute of Technology. http://www.smartech.gatec.edu/

[14] Christensen, B. (2014) Karnofsky Performance Status Scale. http://emedicine.medscape.com/article

[15] CBTRUS (2011) Statistical Report: Primary Brain Tumors in the United States. Central Brain Tumor Registry of the United States, Hinsdale, IL.

[16] Strong, M., Garces, J., Vera, J., Mathkour, M. and Emerson, N. (2015) Brain Tumors: Epidemiology and Current Trends in Treatment. Brain Tumors Neurooncology, 7, 8 .

[17] Parkin, D. and Bray, F. (2005) Global Cancer Statistics. Cancer Journal for Clinicians, 55, 74-108. https://doi.org/10.3322/canjclin.55.2.74

[18] Matsuda, T. and Marugame, T. (2011) Cancer Incidence and Incidence Rates in Japan. Japan Journal for Clinical Oncology, 41, 139-147. https://doi.org/10.1093/jjco/hyq169

[19] McKeran, R. and Thomas, D. (1980) The Clinical Study of Gliomas. Butterworths, London, 194-230.

[20] Krex, D., Klink, B., Hartmann, C., et al. (2007) Long-Term Survival with Glioblastoma Multiforme. Brain, 130, 45-49. https://academic.oup.com/brain/article/130/10/2596/376628

[21] Frei, E. and Canelos, G. (2001) A Critical Factor in Cancer Chemotherapy. American Journal of Medicine, 69, 585-594. https://doi.org/10.1016/0002-9343(80)90472-6

[22] Wara, W. (1985) Radiation Therapy for Brain Tumors. Journal of Neuro-Oncology, 2291-2295.

[23] Greenberg, M. (2010) Handbook of Neurosurgery. Thieme Medical Publishers, New York, Vol. 7, 245-247.

\section{Abbreviations Used in This Article}

GHD: General Hospital Douala.

IV: Intravenous.

CSF: Cero-Spinal Fluid.

CG: Cerebral Glioma.

GBM: Glioblastoma Multiforme.

ICP: Intracranial Pressure.

CT: Computed Tomography.

MRI: Magnetic Resonance Imaging.

WHO: World Health Organization.

USA: United States of America. 